\title{
Jornada de CESN 2526-8236
}

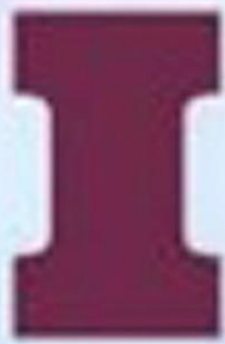

\section{Câncer Ginecológico}
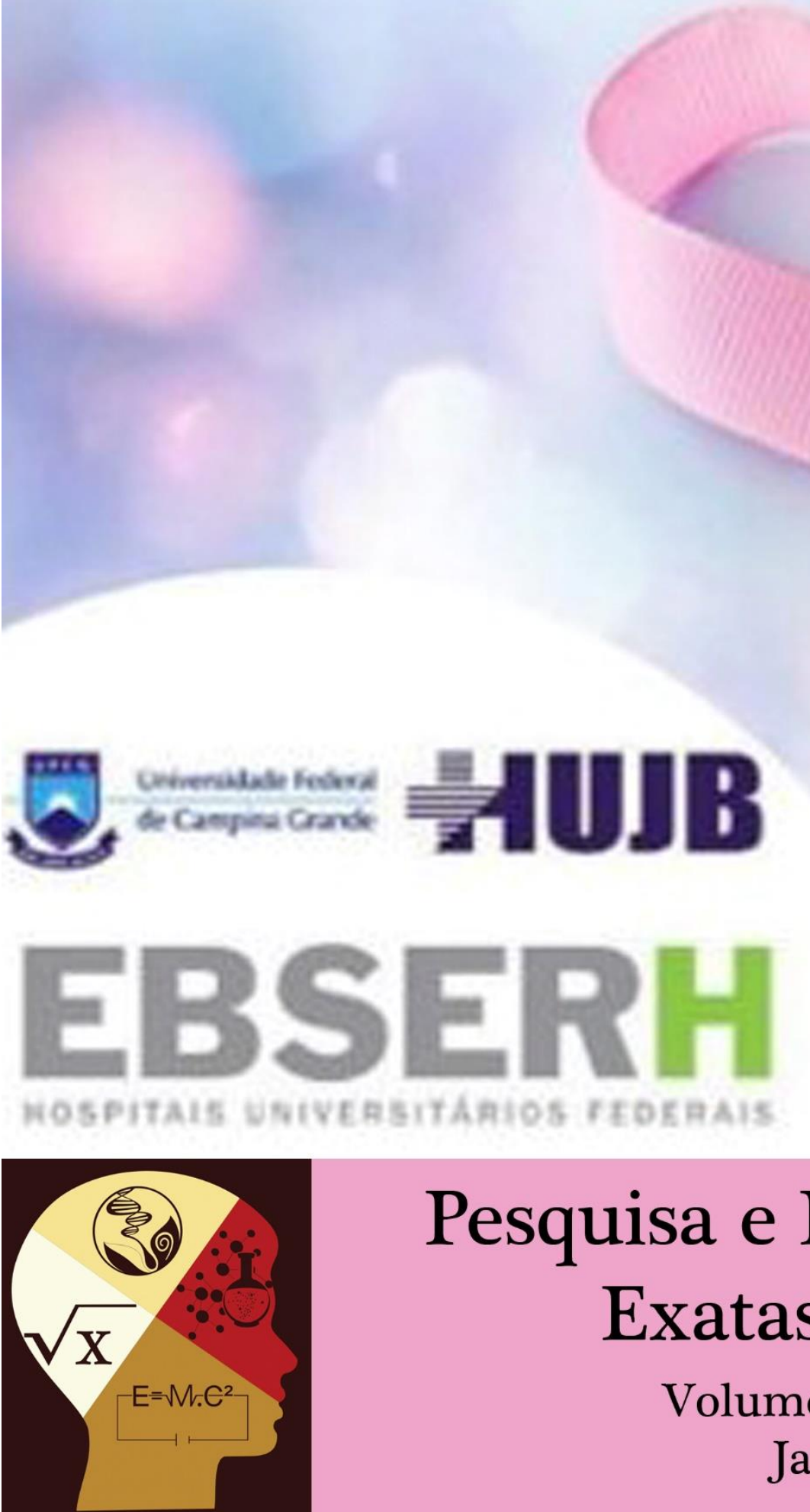

Pesquisa e Ensino em Ciências Exatas e da Natureza

Volume 5 (Edição especial 1)

Janeiro-Fevereiro 


\section{PESQUISA E ENSINO EM CIÊNCIAS EXATAS E DA NATUREZA}

\section{EDITORS-IN-CHIEF}

Dr. Diego Marceli Rocha (UFCG/CFP); Dr. Eudes Leite de Lima (UFCG/CFP); Dr. Ezequiel Fragoso Vieira Leitão (UFCG/CFP); Dr. Heydson Henrique Brito Silva (UFPE); Dr. Silvio Felipe Barbosa de Lima (UFCG/CFP);

\section{EDITORIAL AND SCIENTIFIC BOARD}

Dr. Alessandre P. Colavite (UFPB/CCEN/DSE); Dr. Alexandre Campos (UFCG/CCT/UAF); Dra Albaneide F. Wanderley (UFCG/CFP/UACEN); M. Sc. Arielson dos S. Protázio (UFRB/CCAAB); Dr. Carlos Celestino Rios e Souza (UFPE/CFCH); Dr. Carlos Davidson Pinheiro (UFCG/CFP/UACEN); Dra Claudia Mazza Dias (UFRRJ/IM); Dr. Cleber Ibraim Salimon (UEPB/CCBSA); Dr. Diego Marceli Rocha (UFCG/CFP); Dr. Douglas Fregolente (UFCG/CFP/UACEN); M. Sc. Edilson Leite da Silva (UFCG/CFP/UACEN); Dr. Etielle Barroso de Andrade (IFPI); Dr. Eudes Leite de Lima (UFCG/CFP/UACEN); Dr. Everton Vieira da Silva (UFCG/CFP/UACEN); Dr. Ezequiel Fragoso Vieira Leitão (UFCG/CFP/UACEN); Dra Fabiana F. Q. Freitas (UFCG/CFP/UAENF); Dr. Felipe Wartchow (UFPB/CCEN/DSE); Dr. Fernando Antônio P. da Cunha (UFCG/CFP); Dr. Flavio Almeida Alves Junior (UFPE/CTG); M. Sc. Francisco Carlos P. da Costa (UFCG/CFP/UACEN); Dr. Francisco E. Matos Costa (UFERSA/DECEN); Dr. Francisco José de Andrade (UFCG/CFP/UACEN); M. Sc. Gustavo A. Figueiredo (UFCG/CFP/UACEN); Dr. Heydson Henrique Brito Silva (UFPE); Dr. Hugo da Silva Florentino (UFCG/CFP/UACEN); Dr. João Maria da Silva (UFCG/CFP/UACEN); Dr. José Deomar de Souza Barros (UFCG/CFP/UACEN); Dr. Luciano Leal de Morais Sales (UFCG/CFP/UACEN); Dr. Marcelo Fulgêncio G. Brito (UFS/CGBS/DBI); Dr. Marcio Bernardino Silva (UFPB/CCEN/DSE); Dra Maria do Carmo A. D. Farias (UFCG/CFP/UACV); Dra Marla Ibrahim U. Oliveira (UFS/CCBS/DBI); Dr. Mario Eduardo R. M. Cavalcanti-Mata (UFCG/CTRN); Dr. Martin L. Christoffersen (UFPB/CCEN/DSE); Dr. Miodeli Nogueira Júnior (UFPB/CCEN/DSE); Dr. Pablo Ariel Martinez (UFS/CCBS/DBI); Dr. Paulo Roberto Medeiros (UFCG/CFP/UACEN); Dr. Rafael da Rocha Fortes (URCA/IB/DERM); Dra Raimunda de F. N. Coêlho (UFCG/CFP/UAL); M. Sc. Raul Azevedo (UFCA/CCAB); M. Sc. Rosana Ferreira de Alencar (UFCG/CFP/UACEN); Dr. Samuel Vieira Brito (UFMA/CCAA); Dr. Samuel Cardozo Ribeiro (UFCA); Dr. Saulo Pomponet Oliveira (UFPR/DMAT); Dr. Silvio Felipe Barbosa de Lima (UFCG/CFP/UACEN); Dra Thatyara Freire de Souza (UFERSA/CMPF); Dr. Ticiano Vanderlei de S. Alves (IFPB/CACC); Dr. Udson Santos (UFCG/CFP/UACEN); Dr. Washington L. da Silva Vieira (UFPB/CCEN/DSE);

\section{NATIONAL ASSOCIATE EDITORS}

Dr. Angel Ramon S. Delgado (UFRRJ/ICE/DM); Dra Anne Evelyne F. de Souza (UFPB/CCA/DCV); Dra Carina Scanoni Maia (UFPE/CCB/DHE);
Dr. Carlos Alberto de O. Magalhães Júnior (UEM/CCE); Dr. Carlos Alexandre B. Garcia (UFS/CCET/DQI); Dr. Carlos A. R. Vera-Tudela (UFRRJ/ICE/DM); Dra Daniela Franco Carvalho (UFU/ICB/IB); Dr. Elio Carlos Ricardo (USP/FE); Dra Elisabete Maria Zanin (URI/FURI); Dr. Etielle Barroso de Andrade (IFPI); Dra Fabiana Ferraz Q. Freitas (UFCG/CFP/UAENF); Dr. Fábio Giovanni de Araújo Batista (FCM); M. Sc. Fagno dallino Rolin (UFCG/CFP); Dra Flaviana Jorge de Lima (URCA/DCB); Dr. George Sand Leão Araújo de França (UnB/OS); Dr. Guilherme L. Lucena dos Santos (UFPB/CCA); Dr. Gustavo Benitez Alvarez (UFF/EEIMVR/VCE); M. Sc. Gustavo Lopes Ferreira (IF Goiano); Dr. Isaac Pinheiro dos Santos (UFES/CEUNES); Dra Jacqueline S. S. Cavalcanti (UFRPE/UAST); Dr. João Paulo C. B. da Silva (UFPB/CCEN/DSE); Dr. José Marcos de Castro Nunes (UFBA/IB/DB); Dr. José Ricardo Miras Mermudes (UFRJ/IB/DZ); Dra Laédna Souto Neiva (UFCA/CCT); Dr. Leonardo Cruz da Rosa (UFS/CCAA/DEPAq); Dr. Leonardo Esteves Lopes (UFV/CEDAF); Dra Lindisley Ferreira Gomides (FADIP); Dra Lydia Dayanne Maia Pantoja (UECE/CCS); Dr. Manoel dos Santos Filho (UNEMAT/DBio); Dr. Marcelo Veronesi Fukuda (MZUSP); Dra Maria de Lourdes B. Trindade Galo (UNESP/FCT); Dr. Mauro de Melo Júnior (UFRPE/DB); Dra Michela Borges (UNICAMP/IB/ZUEC); Dr. Paulo Henrique R. Peixoto (UFPE/CAA/NFD); Dra Renata P. Reis (IPJBRJ/Diretoria de Pesquisas); Dra Rita de Cássia M. C. Rodriguez (UFPel/IB/DB); Dr. Rodrigo Fernandes Lira de Holanda (UFRN/CCET); Dr. Rodrigo Giesta Figueiredo (UFES/CCENS/DB); Dr. Rômulo Gil de Luna (UFCG/CCTA/UATA); Dra Rosane Ferreira de Oliveira (UFRRJ/ICE/DM); Dr. Sérgio André F. Azevedo (UFPB/CCEN/DFIS); Dr. Silvânio Silvério Lopes da Costa (UFS); Dra. Suiane Ewerling da Rosa (UFOB); Dr. Ulysses Paulino de Albuquerque (UFPE/CB); Dr. Vinicius Queiroz Araújo (USP/IB); Dr. Wemerson Neves Matias (UFCG/HUJB);

\section{FOREIGN ASSOCIATE EDITORS}

Prof. Fabrizio Scarabino (Udelar/Uruguai); Dr. Jesús Souza Troncoso (UVIGO/FCM/Espanha); Dr. Paulo A. L. F. Coelho (UC/DEC, Portugal); Dr. Pedro Miguel Callapez (UC/FCTUC/Portugal);

\section{ASSISTANT AND PRODUGTION EDITORS}

Sr. Danilo Sousa de Freitas (UFCG/CSTR/UACV); Sr. Erly de Lima Ferreira (UFCG/CFP); Dr. Flavio de Almeida Alves Junior (UFPE/CTG); Dr. Onireves Monteiro de Castro (UFCG/CFP); Dr. Silvio Felipe Barbosa de Lima (UFCG/CFP);

\section{TECHNICAL SUPPORT / GRAPHIC DESIGNER}

Sr. Alejandro Esteweson Santos Faustino Costa (UFPE); Sr. Antonio Lourenço de Araújo Filho (UFCG); Sr. Délio Jackson Dantas Duarte (UFCG); 
- Pesquisa e Ensino em Ciências Exatas e da Natureza is a peer-reviewed, free-access multidisciplinary electronic journal with no publication fees edited by a team of professors of the Academic Unit of Exact and Natural Sciences (UACEN) of the Professor Training Center (CFP) of the Federal University of Campina Grande (UFCG) - Cajazeiras campus - in the state of Paraíba, Brazil.

- The journal is directed primarily to the academic-scientific community and continually receives contributions for the publication of regular editions as well as thematic and/or special editions.

- The aim of the journal is to publish contributions written in Portuguese or English in all fields related to research and teaching in the Exact and Natural Sciences as well as Agrarian Sciences, Biomedical Sciences, Earth Sciences, Education and Teaching, Engineering Sciences, Environmental Sciences, Health Sciences, Humanities and Social Sciences etc., in an annual volume/issue.

- The journal publishes original articles and notes based on scientific research, original articles of a theoretical-methodological nature, literature reviews, book presentations, points of view, news, opinions, experience reports, printing errors, obituaries and editorials.

\section{INDEXED}

- ABEC Brasil;

- Actualidad Iberoamericana

- AGROBASE;

- ASFA;

- Bielefeld Academic Search Engine;

- Biblat;

- Bibliothek: Wissenschaftsparks A. Einstein;

- CAPES;

- CEE Index;

- CIARD;

- C.I.R.C.;

- CiteFactor;

- CLASE;

- Diadorim;

- Dialnet;

- DRJI;

- Eurasian Scientific Journal Index;

- EuroPub;

- General Impact Factor;

- Google Scholar;

- Hochschule Hannover (University of Applied Sciences and Arts);

- Index Copernicus Internacional;

- IRESE;

- JIFACTOR;
- Journals For Free;

- LA Referencia;

- Latindex;

- LivRe;

- MIAR;

- Neliti;

- oasisbr;

- PKP INDEX;

- Publons;

- RCAAP;

- REDIB;

- Repositorio Institucional del CONICET;

- ResearchBib;

- ResearchGate;

- Research Journal Impact Factor;

- ROOTINDEXING;

- Scientific Indexing Services (SIS);

- Scientific Journal Impact Fator;

- SCILIT;

- Sherpa/Romeo;

- Sumários.org;

- University Bibliothek Leipzig;

- Universidad Nacional Autónoma de México;

- Zeitschriften Datenbank Catalogue;

- ZooBank; 


\section{COVER AND CONTENTS}

\section{GIÊNCIAS DA SAÚDE / HEALTH SGIENGES}

Neoplasia maligna da mama: perfil dos óbitos de pacientes no estado

Elinadja Targino do Nascimento

Distribuição da mortalidade por cânceres ginecológicos no estado da

Girleide Santos do Nascimento, Kelvyn Kennedy de Figueiredo Silva, Bruna Braga Dantas

Caracterização de mulheres que não procuraram o resultado do exame PDF Papanicolaou em uma Unidade Básica de Saúde

Uso da estimulação transcraniana por corrente contínua em pacientes PDF com câncer de mama com alterações cognitivas causadas pela 01-07 quimioterapia: revisão integrativa Renata de Lima Martins, Thuanne Karine do Nascimento, Roberto Vinícius Antonino da Costa, Gabriela Brasileiro Campos Mota, Kedma Anne Lima Gomes

Estratégias utilizadas para a prevenção do câncer de colo uterino na PDF atenção primária em saúde: revisão da literatura

Moacir Andrade Ribeiro Filho, Glauberto da Silva Quirino, Mariana Alexandre Gadelha de Lima, Maria do Socorro Lopes Rolim

Avaliação dos resultados do exame papanicolaou não procurados por mulheres de uma Unidade Básica de Saúde no município de Cajazeiras

- Paraíba

Lyvia Maria Fernandes, Francisco Assis Cavalcante Junior, Rogéria Mônica Seixas Xavier de Abreu, Marcos Alan Sousa Barbosa, Marilena Maria de Souza

A cobertura vacinal do HPV no município de Cajazeiras, estado da PDF Paraíba (nordeste do Brasil)

Mariana Alexandre Gadelha de Lima, Alessandra Emilly Pinto de Assis, Irlla Jorrana Bezerra Cavalcante, Moacir Andrade Ribeiro Filho, Symara Abrantes Albuquerque de Oliveira Cabral 